\title{
Is charge quantization exact?
}

There is mild excitement at the prospect of using anti-hydrogen as a means of telling whether the charges of protons and antiprotons differ, and, if so, by how much.

If only the magnitude of the electric charges of the electron and the proton were slightly different, the expansion of the Universe would be accounted for at a stroke: electrostatic repulsion would blow it apart. That is what Einstein suggested in 1924. And the argument can be turned around to infer, from the known expansion of the Universe (the long-standing uncertainty in Hubble's constant notwithstanding) that the charge difference between electrons and protons cannot be large, for that would force the Universe to expand more rapidly than is observed. In other words, there is no obvious reason to doubt what the elementary textbooks say, that the charges of the electron and the proton are equal in magnitude but opposite in sign, so that an intact hydrogen atom is electrically neutral.

What this implies, in turn, is general support for the idea that electric charge is strictly quantized, in units of the elementary charge $\pm e$, where the electric charge of a proton is $+e$ and that of an electron $-e$. Although, when several nucleons are thrown together to make a nucleus, the total mass will be smaller than the sum of the masses of its parts (by the mass equivalent of the binding energy), the total charge is strictly the algebraic sum of the charges of the component nucleons.

The only known exceptions to the rule that electric charge is strictly quantized are the particles called quarks, but they are exceptions of the kind that may be held to prove the rule. Free quarks, with charges of $\pm \frac{1}{3} e$ and $\pm \frac{2}{3} e$, seem not to be capable of independent existence, but only in combinations (hadrons or mesons) whose total charge is either zero or an integral multiple of $e$.

So why should people such as R. J. Hughes (from the Los Alamos National Laboratory) and B. I. Deutsch (from the University of Aarhus) be concerned with the possibility that the quantization rule may not be strictly correct or, more accurately, with an attempt to estimate its precision? Their case (Phys. Rev. Lett. 69, 578-581; 27 July 1992) is that although the equality of the charges of the proton and the electron has now been proved to the hilt, there is nothing like the same precision in the comparison of the electric charges of the corresponding antiparticles, the antiproton and the positron. They plead for measurements with anti-hydrogen to advance the cause.

The stimulation provided by Einstein's argument is easily appreciated. The ratio of the electrostatic and gravitational forces between an electron and a proton, whatever their distance apart, is the huge number $10^{39}$, which is simply another way of saying that gravitational forces are very much weaker than electrostatic forces. But that implies that the expansion of the Universe is enormously sensitive to any difference between the electric charges of the electron and the proton; Hughes and Deutsch quote R. A. Lyttleton and Hermann Bondi for the estimate that a charge difference of $10^{-20} e$ would account for the expansion of the Universe. But the precision of the measured difference is an order of magnitude better than that, or less than $(0.8 \pm 0.8) 10^{-21} e$. Given that the neutron is now known to be electrically neutral to within $10^{-20} \mathrm{e}$, the electrostatic expansion of the Universe looks like a lost cause.

The interest of charge comparisons involving antiparticles, as Hughes and Deutsch explain it, is more subtle and even profound. What constitutional lawyers would no doubt call the enabling equations of antiparticles, Dirac's equation for example, predict that particles and antiparticles will have equal masses. If that is taken as a given, then it should be possible to make high-precision comparison of the charges of electrons and positrons, or of protons and antiprotons, simply by measuring their respective frequencies of revolution (the cyclotron frequency) in a suitable magnetic field, which is one of the everyday by-products of high-energy physics. But who says that the enabling equations are exact? Hughes and Deutsch argue that that amounts to the assumption of CPT invariance, or that the equations of motion for particles/antiparticles are invariant with respect to charge inversion, parity (or the inversion of space through an arbitrary origin of coordinates) and time reversal - a simple but not necessarily correct assumption about the Universe on a microscopic scale.

So how to compare the electric charges of particles and antiparticles without supposing that their masses are intrinsically equal? Strictly, measurements of the cyclotron frequency are measurements of the ratio of charge and mass, e/m. Plainly an independent measurement is needed if differences of $e$ are to be separately determined. Hughes and Deutsch argue that the best source of further information must be a spectroscopic measurement of an exotic atomic state in which one of the constituents is an antiparticle.

The most obvious candidate is positronium, the atom whose constituents are an electron and a positron. This has a ground state and excited states, just as hydrogen atoms do, but the energy levels are differently spaced because the particles have different masses. Technically, the Rydberg constant for positronium is almost exactly half that for hydrogen. So, given a cyclotron comparison and a measurement of a known energy level of positronium, it should be possible to put a limit on the difference of charge.

There are data in the literature sufficient to pin down the difference of the electric charges of the electron and the positron. The cyclotron frequencies of the two particles are known to differ by one part in $10^{8}$, while the respective Rydberg constants are in a ratio that differs from unity by less than $4 \times 10^{-8}$. There is a prospect that each of the component measurements may be made three orders of magnitude more precise, which would reveal differences of the order of $10^{-11}$ in the two charges. That will still be some way short of the precision with which the charges of the electron and the proton have been compared, but will no doubt not be sniffed at.

The comparison of the charges of the proton and antiproton is more difficult. The analogue of positronium for these particles is the bound state of a proton and antiproton, for which the available mass measurement is much less certain. Hughes and Deutsch argue that the precision of the comparison using data now available is merely two parts in 100,000 , despite the much greater precision of the cyclotron measurements themselves.

So how to make progress? That is where anti-hydrogen comes in. The idea is that isolated atoms of anti-hydrogen would be relatively much more stable than those of positronium (whose constituents can mutually annihilate each other). Hughes and Deutsch calculate that it should be possible to measure the Rydberg constant of antihydrogen to one part in $10^{15}$, and that a suitable cyclotron comparison of proton and antiproton and electron and positron would pin down the difference of charge of proton and antiproton to one part in $10^{11}$.

But first catch some anti-hydrogen. Nobody has made a single atom yet, unless by accident. The ideal will be to prepare a handful of atoms in a suitably cooled ion trap, holding them there long enough for spectroscopy to be possible. It will be interesting to see which laboratory is the first in the field. While the search goes on, the doctrine of CPT invariance lacks underpinning, but at a level of precision unlikely to keep people awake at night.

John Maddox 